REGULARITY AND OTHER ASPECTS

OF THE NAVIER-STOKES EQUATIONS

BANACH CENTER PUBLICATIONS, VOLUME 70

INSTITUTE OF MATHEMATICS

POLISH ACADEMY OF SCIENCES

WARSZAWA 2005

\title{
THE LERAY PROBLEM FOR 2D INHOMOGENEOUS FLUIDS
}

\author{
FARID AMMAR-KHODJA \\ Département de Mathématiques \&S UMR 6623, Université de Franche-Comté \\ 16, route de Gray, 25030 Besançon Cedex, France \\ E-mail: ammar@math.univ-fcomte.fr \\ MARCELO M. SANTOS \\ Departamento de Matemática, IMECC-UNICAMP \\ Cx. Postal 6065, 13.081-970 Campinas, SP, Brazil \\ E-mail:msantos@ime.unicamp.br
}

\begin{abstract}
We formulate the Leray problem for inhomogeneous fluids in two dimensions and outline the proof of the existence of a solution. There are two kinds of results depending on whether the given value for the density is a continuous function or only an $L^{\infty}$ function. In the former case, the given densities are attained in the sense of uniform convergence and in the latter with respect to weak-* convergence.
\end{abstract}

1. Introduction. The classical so-called Leray problem consists of finding a solution for the incompressible stationary Navier-Stokes equations with a constant density, in a domain with unbounded straight channels, such that the velocity of the fluid in each channel tends to a given Poiseuille flow in the end of the channel. A Poiseuille flow is a solution of the incompressible stationary Navier-Stokes equations in a straight channel that is parallel to the walls of the channel and satisfies the nonslip boundary condition, i.e. vanishes at the boundary of the channel; see (5) below. The Leray problem seems to have been proposed, in the 1950s, by Jean Leray to Olga A. Ladyzhenskaya, cf. [1, p. 476]. Despite the effort of brilliant mathematicians, see e.g. [6], up to now its solution is known only in the case of Poiseuille flows with small fluxes, a result due to Charles J. Amick [1, Theorem 3.8]. Not surprisingly, the main difficulty in solving the problem is to deal with the nonlinear term in the Navier-Stokes equations. This difficulty is overcome by seeking

2000 Mathematics Subject Classification: 35Q30, 76D05.

Key words and phrases: stationary Navier-Stokes equations, incompressible flow, inhomogeneous fluid, Leray problem, discontinuous density.

The paper is in final form and no version of it will be published elsewhere. 
a solution with the velocity field $\mathbf{v}$ in the form $\mathbf{v}=\mathbf{u}+\mathbf{a}$, for a new unknown $\mathbf{u}$, where $\mathbf{a}$ is a suitable extension of the given Poiseuille flows. It turns out that the nonlinear term can be estimated by the fluxes of the Poiseuille flows [1], thus the result comes about under the restriction that these fluxes are small, in comparison with the viscosity of the fluid. In the case of inhomogeneous fluids, i.e. fluids with a nonconstant density, besides the given values for the fluid velocity in the ends of the domain, we prescribe values for the density in the 'incoming channels' (i.e. in the channels where the fluid is incoming). In this situation additional problem arise, as for instance, proving that the prescribed values are in some sense attained.

In the case of a continuous given density, we prove the existence of a solution such that the density attains the given value in the supremum norm. In the case of a given $L^{\infty}$ density, we present a new approach that permits to pass from smooth-density solutions to $L^{\infty}$-density solutions. The result for smooth-density solutions is based on the streamline formulation, an approach strictly limited to the two dimensional case. In fact, the smooth density $\rho$ is of the form $\rho=\omega(\psi)$ where $\psi$ is the streamline function, i.e. $\nabla^{\perp} \psi=\mathbf{v}$ $\left(\nabla^{\perp} \psi:=\left(-\partial_{y} \psi, \partial_{x} \psi\right)\right)$, and $\omega$ is some scalar function connected to the given values for the density and for the velocity vector field; cf. [2, 7]. In the case of a $L^{\infty}$-density, the composition $\omega(\psi)$ is for our purpose meaningless, since for a discontinuous $\omega$ it may not yield a measurable function.

For this reason, to obtain a $L^{\infty}$ solution, we regularize the data and take appropriate associated scalar functions $\omega^{\epsilon}, \epsilon>0$. Then we introduce approximating solutions $\rho^{\epsilon}=$ $\omega^{\epsilon}\left(\psi^{\epsilon}\right), \mathbf{v}^{\epsilon}=\nabla^{\perp} \psi^{\epsilon}$, and we are able to pass to the limit as $\epsilon$ tends to zero due to careful (with respect to $\epsilon$ ) uniform estimates derived for the smooth case. An important step in the arguments is to attain the given value for the density, which is posed at the end of the channel where the fluid is incoming, i.e. at $x=-\infty$ in the description of the domain we give below. Here we have a special difficulty, essentially because the density is only in $L^{\infty}$ and we have to deal with the double limit of taking $\epsilon$ tending to zero and $x$ tending to minus infinity. To overcome this difficulty we use the weak formulation of the 'transport equation' $\nabla \cdot(\rho \mathbf{v})=0$ (the stationary equation of conservation of mass), in which the given density at minus infinity is taken into account, and choose special test functions. In fact, we do not get exactly a desired weak formulation. Instead, we get an approximate one, but with an error term that decays exponentially at minus infinity. This is due to the exponential decay of the approximated velocity $\mathbf{v}^{\epsilon}$ to the Poiseuille flow, uniformly with respect to $\epsilon$.

Now, we describe our problem more precisely. The fluid fills a domain $\Omega \subset \mathbb{R}^{2}$ that is simply-connected, it has a smooth unbounded boundary $\Gamma$ and it is the union of three disjoint sets, $\Omega=\Omega_{-} \cup \Omega_{0} \cup \Omega_{+}$, such that $\Omega_{0}$ is bounded and, in possibly different coordinate systems, $\Omega_{-}=\left\{(x, y) \in \mathbb{R}^{2}: x<0, y \in \Sigma_{-}\right\}$and $\Omega_{+}=\left\{(x, y) \in \mathbb{R}^{2}: x>\right.$ $\left.0, y \in \Sigma_{+}\right\}$, with $\Sigma_{ \pm}=\left(-d_{ \pm}, d_{ \pm}\right)$for arbitrarily given constants $d_{ \pm}>0$. That is, $\Omega_{ \pm}$ is given as the image by some affine transformation of the strip $\tilde{\Omega}_{ \pm}=\left\{(x, y) \in \mathbb{R}^{2}\right.$ : $\left.\pm x>0, y \in \Sigma_{ \pm}\right\}$. The open intervals $\Sigma_{ \pm}$can be taken as the same interval $(-1,1)$, and in this case we can write $\tilde{\Omega}_{ \pm}= \pm(0, \infty) \times(-1,1)$. For sake of simplicity we will consider the standard situation $\Omega_{ \pm}= \pm[1, \infty) \times(-1,1)$ and $\Omega=\Omega_{-} \cup \Omega_{0} \cup \Omega_{+}$. The sets $\Omega_{ \pm}$are thought as being the channels. This kind of domain was introduced by Amick, which he 
called an admissible domain [1, Definition 1.1]. The Navier-Stokes equations describing a stationary inhomogeneous incompressible fluid in $\Omega$ are the following:

$$
\left\{\begin{array}{c}
\nu \Delta \mathbf{v}=\rho(\mathbf{v} \cdot \nabla) \mathbf{v}+\nabla p \\
\nabla \cdot \mathbf{v}=0, \quad \nabla \cdot(\rho \mathbf{v})=0 .
\end{array}\right.
$$

Here, $\rho, \mathbf{v}=\left(v_{1}, v_{2}\right), p$, and $\nu$ are, respectively, the mass density, the velocity, the pressure and the given constant viscosity of the fluid. The first equation represents the conservation of momentum and the second and third equations represent the incompressibility of the fluid and the conservation of mass, respectively.

The velocity field $\mathbf{v}$ is assumed to be parallel at far distances in each straight channel and, incoming in $\Omega_{-}$and outgoing in $\Omega_{+}$. Since the conservation of mass equation is a 'transport equation' for the density $\rho$, with the transport vector given by $\mathbf{v}$, it is natural to give the density only at the end of the channel where the fluid is incoming, i.e. in $\Omega_{-}$. Besides, we assume that the fluid does not slip on the boundary $\Gamma$ of $\Omega$, so the vector field $\mathbf{v}$ in each channel $\Omega_{ \pm}$is of Poiseuille type. Thus, coupled with the systems of equation (1), we have the following boundary conditions:

$$
\mathbf{v}=0 \quad \text { on } \Gamma
$$

(nonslip boundary condition)

$$
\lim _{ \pm x \rightarrow \infty} \mathbf{v}=\mathbf{v}_{ \pm}
$$

and

$$
\lim _{x \rightarrow-\infty} \rho=\rho_{-}
$$

where $\mathbf{v}_{ \pm}$is a given Poiseuille flow in $\Omega_{ \pm}$(see (5) below), and $\rho_{-}$is a given function in $C_{b}\left(\Sigma_{-}\right)$or $L^{\infty}\left(\Sigma_{-}\right)$. If $X$ is a topological space, $C_{b}(X)$ will denote the space of bounded and continuous functions defined on $\Omega$, endowed with the supremum norm $\|f\|_{C_{b}(X)}=$ $\sup _{x \in X}|f(x)|$.

To state our main results (see Theorems 1 and 2 below), we need some more notations. First, let

$$
\alpha_{ \pm}=\int_{\Sigma_{ \pm}(x)} \mathbf{v}_{ \pm} \cdot \mathbf{n}_{ \pm}
$$

(the flux of the Poiseuille flow $\mathbf{v}_{ \pm}$in $\left.\Omega_{ \pm}\right)$where $\mathbf{n}_{ \pm}=( \pm 1,0)$ is the unit normal to $\Sigma_{ \pm}(x)=\{x\} \times(-1,1)$ (the cross section of $\Omega_{ \pm}$at ' $x$ '). More precisely, we have

$$
\mathbf{v}_{ \pm}=\mathbf{v}_{ \pm}(y)=\left(\theta_{ \pm}(y), 0\right) \text { for } \theta_{ \pm}(y)= \pm \frac{3}{4} \alpha_{ \pm}\left(1-y^{2}\right), \quad y \in \Sigma_{ \pm}=(-1,1)
$$

cf. [1, p. 485]. Because the incompressibility equation $\nabla \cdot \mathbf{v}=0$ and the Divergence Theorem, we assume the compatibility condition $\alpha_{-}+\alpha_{+}=0$, i.e. $\alpha_{+}=-\alpha_{-}$. We assume also that $\alpha_{-}$is a strictly negative real number (and so $\alpha_{+}>0$ ), i.e. the velocity field $\mathbf{v}_{-}$is incoming in $\Omega_{-}$and $\mathbf{v}_{+}$is outgoing in $\Omega_{+}$. More generally, the flux of a vector velocity field $\mathbf{v}$ through $\Sigma_{ \pm}(x)$ is given by $\int_{\Sigma_{ \pm}(x)} \mathbf{v} \cdot \mathbf{n}_{ \pm}$. Similarly, the flux of the momentum $\rho \mathbf{v}$ through $\Sigma_{ \pm}(x)$ is given by $\int_{\Sigma_{ \pm}(x)} \rho \mathbf{v} \cdot \mathbf{n}_{ \pm}$.

Let $\mathbf{H}_{k, \text { loc }}(\bar{\Omega})$ be the space of vector fields $\mathbf{v}$ in $\Omega$ such that $\mathbf{v}$ belongs to the Sobolev space $W^{k, 2}\left(\Omega^{\prime}\right)$, for any bounded open subset $\Omega^{\prime}$ of $\Omega, \mathbf{v}$ is divergence free, i.e. $\nabla \cdot \mathbf{v}=0$, and its derivatives up to order $k-1$ have vanishing trace on $\Gamma$. Let also $\mathcal{V}$ be the space 
of the vector fields $\boldsymbol{\Phi}$ in $C_{0}^{\infty}(\Omega)$ (the underscript ' 0 ' stands for compact support, i.e. the support set of $\boldsymbol{\Phi}$ is a compact set contained in $\Omega$ ) and $\boldsymbol{\Phi}$ is divergence free, i.e. $\nabla \cdot \boldsymbol{\Phi}=0$. Finally, let $l=\left\|\rho_{-}\right\|_{L^{\infty}\left(\Sigma_{-}\right)}\left(l=\left\|\rho_{-}\right\|_{C_{b}\left(\Sigma_{-}\right)}\right.$if $\left.\rho_{-} \in C_{b}\left(\Sigma_{-}\right)\right)$. Our main results are the following theorems.

TheOREM 1. Assume that $\rho_{-} \in C_{b}\left(\Sigma_{-}\right)$. Then there is a constant $c=c(\Omega)>0$ such that for $c\left|\alpha_{-}\right| l<\nu$, the problem (1)-(4) has a weak solution $(\rho, \mathbf{v}) \in C_{b}(\Omega) \times \mathbf{H}_{1, l o c}(\bar{\Omega})$, in the following sense:

$i$.

$$
\nu \int_{\Omega} \nabla \mathbf{v} \cdot \nabla \boldsymbol{\Phi} d x=\int_{\Omega} \rho(\mathbf{v} \cdot \nabla \boldsymbol{\Phi}) \cdot \mathbf{v} d x
$$

for all $\boldsymbol{\Phi}=\left(\Phi_{1}, \Phi_{2}\right)$ in $\mathcal{V}$, where $\nabla \mathbf{v} \cdot \nabla \boldsymbol{\Phi}:=\nabla v_{1} \cdot \nabla \Phi_{1}+\nabla v_{2} \cdot \nabla \Phi_{2}$ and $\mathbf{v} \cdot \nabla \boldsymbol{\Phi}:=\left(\mathbf{v} \cdot \nabla \Phi_{1}, \mathbf{v} \cdot \nabla \Phi_{2}\right)$,

$i i$.

$$
\int_{\Omega} \rho \mathbf{v} \cdot \nabla \varphi d x=0 \quad \text { for all } \varphi \text { in } C_{0}^{\infty}(\Omega)
$$

iii.

$$
\mathbf{v}-\mathbf{v}_{ \pm} \in W^{2,2}\left(\Omega_{t}^{c}\right), \quad \text { for some } t>0,
$$

where $\Omega_{t}^{c}:=\Omega_{-, t}^{c} \cup \Omega_{+, t}^{c}, \quad \Omega_{ \pm, t}^{c}:=\left\{(x, y) \in \Omega_{ \pm} ; \pm x>t\right\} ;$ and $i v$.

$$
\lim _{x \rightarrow-\infty}\left\|\rho(x, \cdot)-\rho_{-}\right\|_{C_{b}\left(\Sigma_{-}\right)}=0 .
$$

Furthermore, the flux of $\mathbf{v}$ in $\Omega_{ \pm}$is equal to $\alpha_{ \pm}$and the flux $\beta_{ \pm}$of the momentum $\rho \mathbf{v}$ in $\Omega_{ \pm}$can be written as

$$
\beta_{ \pm}= \pm \int_{\alpha_{-}}^{0} \rho_{-}\left(\psi_{-}^{-1}(s)\right) d s
$$

where $\psi$ and $\psi_{-}$are stream functions associated with $\mathbf{v}$ and $\mathbf{v}_{-}$, respectively, i.e. $\nabla^{\perp} \psi=$ $\mathbf{v}$ and $\psi_{-}{ }^{\prime}=-\theta_{-}$; more precisely, we take $\psi(x, y)=-\left( \pm \int_{-1}^{y} v_{1}(x, \tau) d \tau\right)$ in $\Omega_{ \pm}$and $\psi_{-}(y)=-\int_{-1}^{y} \theta_{-}(\tau) d \tau$ in $\Sigma_{ \pm}$. Finally, we have $\|\rho\|_{C_{b}(\Omega)} \leq l$ and

$$
\left\|\nabla\left(\mathbf{v}-\mathbf{v}_{ \pm}\right)\right\|_{L^{2}\left(\Omega_{ \pm}\right)}+\|\nabla \mathbf{v}\|_{L^{2}\left(\Omega_{0}\right)} \leq C\left|\alpha_{ \pm}\right|\left(1+\frac{\nu+\left|\alpha_{ \pm}\right| l}{\nu-c\left|\alpha_{ \pm}\right| l}\right)
$$

for some other constant $C=C(\Omega)$.

TheOrem 2. Assume that $\rho_{-} \in L^{\infty}\left(\Sigma_{-}\right)$. Then there is a constant $c=c(\Omega)>0$ such that for $c\left|\alpha_{-}\right| l<\nu$, the problem (1)-(4) has a weak solution $(\rho, \mathbf{v}) \in L^{\infty}(\Omega) \times \mathbf{H}_{1, l o c}(\bar{\Omega})$, in the sense that it satisfies $i$. to iii. of Theorem 1 and additionally

$i v$.

$$
*_{x \rightarrow-\infty, a . e .} \rho(x, \cdot)=\rho_{-},
$$

where $*-\lim _{x \rightarrow-\infty, a . e .}$ denotes the limit in the weak-* topology of $L^{\infty}\left(\Sigma_{ \pm}\right)$, with $x$ tending to $-\infty$ except for a set of zero Lebesgue measure.

Equations (6) and (7) are just the weak formulations (in the sense of distributions) of the conservation of momentum and mass equations, respectively, i.e. just multiply these equations by the test functions indicated in (6) and (7) and formally integrate by parts. 
In equation (6) the pressure $p$ is canceled out because the (vector valued) test functions $\boldsymbol{\Phi}$ are divergence free. It is classical that we can recover the pressure from (6); see e.g. [8, Propositions I.1.1 and I.1.2, p. 14]. The incompressibility equation is inserted in the space $\mathbf{H}_{1, l o c}(\bar{\Omega})$. Condition (8) implies that $\mathbf{v} \in C_{b}\left(\Omega_{t}^{c}\right)$ and

$$
\lim _{ \pm x \rightarrow \infty}\left\|\mathbf{v}(x, \cdot)-\mathbf{v}_{ \pm}\right\|_{C_{b}\left(\Sigma_{ \pm}\right)}=0 .
$$

Indeed, since $\Omega_{ \pm, t}^{c}$ is bounded in one direction, from the Sobolev Imbedding Theorem, we have $\mathbf{v}-\mathbf{v}_{ \pm} \in C_{b}\left(\Omega_{ \pm, t}^{c}\right)$ and there is a constant $k$, independent of $|x|>t+1$, such that

$$
\left\|\mathbf{v}(x, \cdot)-\mathbf{v}_{ \pm}\right\|_{C_{b}\left(\Sigma_{ \pm}\right)} \leq\left\|\mathbf{v}-\mathbf{v}_{ \pm}\right\|_{C_{b}\left(\Omega_{ \pm,|x|-1}^{c}\right)} \leq k\left\|\mathbf{v}-\mathbf{v}_{ \pm}\right\|_{W^{2,2}\left(\Omega_{ \pm,|x|-1}^{c}\right)}
$$

thus $\lim _{ \pm x \rightarrow \infty}\left\|\mathbf{v}(x, \cdot)-\mathbf{v}_{ \pm}\right\|_{C_{b}\left(\Sigma_{ \pm}\right)} \leq k \lim _{ \pm x \rightarrow \infty}\left\|\mathbf{v}-\mathbf{v}_{ \pm}\right\|_{W^{2,2}\left(\Omega_{ \pm,|x|-1}^{c}\right)}=0$. Equation (12) says that the given density value $\rho_{-}$at the end of the incoming channel $\Omega_{-}$is attained 'in average' almost everywhere, i.e. there is a Lebesgue measurable set $E \subset \Sigma_{-}$ with zero Lebesgue measure such that

$$
\lim _{\substack{x \rightarrow-\infty \\ x \in \Sigma_{-} / E}} \int_{\Sigma_{-}} \rho(x, y) \xi(y) d y=\int_{\Sigma_{-}} \rho_{-}(y) \xi(y) d y
$$

for all $\xi \in L^{1}\left(\Sigma_{-}\right)$.

In the next sections we outline the proofs of Amick's theorem for the classical Leray problem and of Theorems 1 and 2 .

2. The classical Leray problem. In this section we outline the proof of Amick's theorem for the classical Leray problem. Amick's theorem states that the problem (1)-(3) with $\rho \equiv 1$ (constant density) has a weak solution whenever the flux $\alpha_{-}\left(\alpha_{+}\right)$is sufficiently small. The proof starts with the variational formulation (6) where, as we mentioned above, $\mathbf{v}$ is sought in the form $\mathbf{v}=\mathbf{u}+\mathbf{a}$ for a suitable extension a of the given Poiseuille flows; see (3) and (5). This extension belongs to $\mathbf{H}_{1, l o c}(\bar{\Omega})$, coincides with the Poiseuille flow in $\Omega_{ \pm, t}^{c}$ for some large $t$, and in $\Omega_{t}:=\Omega_{-, t} \cup \Omega_{0} \cup \Omega_{+, t}, \Omega_{ \pm, t}:=\left\{(x, y) \in \Omega_{ \pm} ; \pm x<t\right\}$, it satisfies the estimate

$$
\|\nabla \mathbf{a}\|_{L^{2}\left(\Omega_{t}\right)} \leq c\left|\alpha_{-}\right|
$$

for some positive constant $c=c(\Omega)$; see $[1, \S 3.1]$ or [3, Lemma XI.3.1]. The existence of the new unknown $\mathbf{u}$ satisfying

$$
\nu \int_{\Omega} \nabla(\mathbf{u}+\mathbf{a}) \cdot \nabla \boldsymbol{\Phi} d x=\int_{\Omega}((\mathbf{u}+\mathbf{a}) \cdot \nabla \boldsymbol{\Phi}) \cdot(\mathbf{u}+\mathbf{a}) d x
$$

for all $\boldsymbol{\Phi} \in \mathcal{V}$ can be obtained, for instance, in the space $\mathbf{V}$ - the closure of $\mathcal{V}$ in the Dirichlet norm $\|\nabla \mathbf{u}\|_{L^{2}(\Omega)}$-by Galerkin method [3, 4] or Leray-Schauder principle (cf. $[2]$ ), once we have a priori estimate for $\mathbf{u}$ in $\mathbf{V}$. To obtain a priori estimate for $\mathbf{u}$ in $\mathbf{V}$ we multiply the conservation of momentum equation by $\mathbf{u}$ and integrate by parts (alternately, we can take $\boldsymbol{\Phi}=\mathbf{u}$ in (14)). Thus we have

$$
\nu\|\nabla \mathbf{u}\|_{L^{2}(\Omega)}^{2}=-\nu \int_{\Omega_{t}} \nabla \mathbf{a} \cdot \nabla \mathbf{u}+\int_{\Omega}(\mathbf{u} \cdot \nabla) \mathbf{u} \cdot \mathbf{a}+\int_{\Omega}(\mathbf{a} \cdot \nabla) \mathbf{u} \cdot \mathbf{a}
$$


To control the 'quadratic' term on the left hand side of (15), we need to estimate the three terms on the right hand side. As we pointed out in the Introduction, the difficult term is the nonlinear one, here the second term on the right hand side of (15), since it is also quadratic with respect to $\mathbf{u}$. It is this term that imposes the restriction regarding the size of the flux $\alpha_{-}$for the existence of a solution to the problem (1)-(3). To estimate it, we separate the integral in two parts, one with the integral over $\Omega_{t}$ and the other, over $\Omega_{t}^{c}=$ $\Omega_{-, t}^{c} \cup \Omega_{+, t}^{c}$. The first integral is bounded by $c\left|\alpha_{-}\right||| \mathbf{u} \|_{L^{2}\left(\Omega_{t}\right)}^{2}$ in virtue of (13), Hölder's inequality and Sobolev type inequality, i.e. $\left|\int_{\Omega_{t}}(\mathbf{u} \cdot \nabla) \mathbf{u} \cdot \mathbf{a}\right| \leq\|\mathbf{u a}\|_{L^{2}\left(\Omega_{t}\right)}|| \nabla \mathbf{u} \|_{L^{2}\left(\Omega_{t}\right)} \leq$ $\|\mathbf{a}\|_{L^{4}\left(\Omega_{t}\right)}|| \mathbf{u}\left\|_{L^{4}\left(\Omega_{t}\right)}|| \nabla \mathbf{u}\right\|_{L^{2}\left(\Omega_{t}\right)} \leq c|| \nabla \mathbf{a}\left\|_{L^{2}\left(\Omega_{t}\right)}|| \nabla \mathbf{u}\right\|_{L^{2}\left(\Omega_{t}\right)}^{2}$, where from now on $c$ denotes some positive constant depending only on $\Omega$. For the second, we estimate $\|\mathbf{u a}\|_{L^{2}\left(\Omega_{ \pm, t}^{c}\right)}$ using Fubini's theorem applied on $\Omega_{ \pm, t}^{c}$ and Hölder and Sobolev type inequalities applied on $\Sigma_{ \pm}$as follows.

$$
\begin{aligned}
\int_{\Omega_{ \pm, t}^{c}}|\mathbf{u}|^{2}|\mathbf{a}|^{2} & = \pm \int_{ \pm t}^{ \pm \infty} \int_{\Sigma_{ \pm}}|\mathbf{u}|^{2}|\mathbf{a}|^{2} \leq \pm \int_{ \pm t}^{ \pm \infty}\|\mathbf{u}\|_{L^{4}\left(\Sigma_{ \pm}\right)}^{2}\|\mathbf{a}\|_{L^{4}\left(\Sigma_{ \pm}\right)}^{2} \\
& \leq \pm c \int_{ \pm t}^{ \pm \infty}\|\nabla \mathbf{u}\|_{L^{2}\left(\Sigma_{ \pm}\right)}^{2}\|\nabla \mathbf{a}\|_{L^{2}\left(\Sigma_{ \pm}\right)}^{2} .
\end{aligned}
$$

Next we use that $\|\nabla \mathbf{a}\|_{L^{2}\left(\Sigma_{ \pm}\right)}$is proportional to the flux $\alpha_{-}$, which can be seen directly from (5). Then, the second term on the RHS of (15) is estimated by $c\left|\alpha_{-}\right||| \nabla \mathbf{u} \|_{L^{2}(\Omega)}^{2}$. The other terms on the RHS of (15) can be estimated by $c\left|\alpha_{-}\right| \mid \nabla \mathbf{u} \|_{L^{2}(\Omega)}$. Therefore we obtain that $\|\nabla \mathbf{u}\|_{L^{2}(\Omega)}^{2}$ is bounded by some constant independent of $\mathbf{u}$ if $c\left|\alpha_{-}\right|<<\nu$. For a rigorous proof we refer to [1] or [3, Chapter XI].

3. The density-dependent Leray problem: Continuous case. In this section we outline the proof of Theorem 1. For a rigorous and complete proof and more details we refer the reader to [4].

Our approach depends on the streamline formulation $\rho=\omega(\psi), \mathbf{v}=\nabla^{\perp} \psi$, where $\omega \in C_{b}(\mathbb{R})$ is connected to the data $\mathbf{v}_{-}$and $\rho_{-}$, essentially by the formula $\omega=\rho_{-} \circ \psi_{-}^{-1}$. This is a natural choice because we expect that at $-\infty$ we have $\rho=\rho_{-}$and $\psi=\psi_{-}$ so $\omega\left(\psi_{-}(y)\right)$ should be equal to $\rho_{-}(y)$ for all $y \in \Sigma_{-}$. More precisely, $\omega$ is a function in $C_{b}(\mathbb{R})$ satisfying

$$
\omega(s)=\rho_{-}\left(\psi_{-}^{-1}(s)\right), \quad \forall s \in \psi_{-}\left(\Sigma_{-}\right) .
$$

We recall that $\psi_{-}$is the streamline function of $\mathbf{v}_{-}$defined in Theorem 1 , i.e. $\psi_{-}(y):=$ $-\int_{-1}^{y} \theta(\tau) d \tau$. We note that $\psi_{-}$is a monotonic function for $y \in \Sigma_{-}$, thus (16) defines $\omega$ in a unique way and $\psi_{-}\left(\Sigma_{-}\right)$is the interval $\left(0,-\alpha_{-}\right)$. To have the condition $\|\rho\|_{C_{b}(\Omega)} \leq l$ satisfied it is enough to extend $\omega$ in $C_{b}(\mathbb{R})$ outside the interval $\left(0,-_{-}\right)$ such that $\|\omega\|_{C_{b}(\mathbb{R})} \leq l$. For a $\omega$ satisfying the above conditions we look for a solution $\mathbf{v}=\nabla^{\perp} \psi$ of (6) with $\rho=\omega(\psi)$ and $\mathbf{v}=\mathbf{u}+\mathbf{a}=\nabla^{\perp} \psi$ where $\mathbf{a}$ is as in the previous section. ( $\psi$ will solve the nonlinear equation for the biharmonic operator $\Delta^{2}, \nu \Delta^{2} \psi=\operatorname{curl}\left(\omega(\psi)\left(\nabla^{\perp} \psi \cdot \nabla\right) \nabla^{\perp} \psi\right)$, which can be seen by applying the operator curl $=\partial_{y}-\partial_{x}$ to the conservation of momentum equation.) Indeed, it is possible to prove the following theorem by Leray-Schauder principle (cf. [2]) or Galerkin method [4]. 
Theorem 3. Given any $\omega$ in $C_{b}(\mathbb{R})$, there is a constant $c=c(\Omega)>0$ such that for $c\left|\alpha_{-}\right||| \omega \|_{C_{b}(\mathbb{R})}<\nu$, the problem

$$
\nu \int_{\Omega} \nabla(\mathbf{u}+\mathbf{a}) \cdot \nabla \mathbf{\Phi} d x=\int_{\Omega} \omega(\psi)((\mathbf{u}+\mathbf{a}) \cdot \nabla \boldsymbol{\Phi}) \cdot(\mathbf{u}+\mathbf{a}) d x, \forall \mathbf{\Phi} \in \mathcal{V}
$$

has a solution $\psi \in W_{\text {loc }}^{2,2}(\Omega)$, where $\mathbf{u}+\mathbf{a}=\nabla^{\perp} \psi, \mathbf{u} \in \mathbf{V}$.

To prove this theorem, proceeding as in the previous section, we can estimate the nonlinear term $\int_{\Omega} \omega(\psi)(\mathbf{u} \cdot \nabla) \mathbf{u} \cdot \mathbf{a}$ from above by $c\left|\alpha_{-}\right||| \omega\left\|_{C_{b}(\mathbb{R})}|| \nabla \mathbf{u}\right\|_{L^{2}(\Omega)}^{2}$. Then any sequence $\left(\psi_{k}\right), \psi_{k}=\mathbf{u}_{k}+\mathbf{a}$, of approximating solution to (17) is bounded in $W^{2,2}\left(\Omega^{\prime}\right)$ where $\Omega^{\prime}$ is an arbitrary bounded open subset of $\Omega$. Thus, from the Sobolev imbedding theorem, we have that $\left(\psi_{k}\right)$ converges to some $\psi$ in $C_{b}\left(\Omega^{\prime}\right)$. Since $\omega$ is fixed and continuous, $\omega\left(\psi_{k}\right)$ converges to $\omega(\psi)$, also in $C_{b}\left(\Omega^{\prime}\right)$. With these estimates it is possible to pass to the limit in (17) to obtain an exact solution from approximating solutions.

Since $\psi \in W_{l o c}^{2,2}(\bar{\Omega})$, the pair $(\rho, \mathbf{v})=\left(\omega(\psi), \nabla^{\perp} \psi\right)$ belongs to $C_{b}(\Omega) \times \mathbf{H}_{1, l o c}(\bar{\Omega})$ and it is obvious from (17) that it satisfies (6) in Theorem 1. The condition (7) is certainly true in the case of $\omega \in C^{1}(\mathbb{R})$. In the case of $\omega$ only continuous, we first consider (17) with approximating smooth functions $\omega^{\epsilon}$ uniformly bounded in $C_{b}(\mathbb{R})$ with respect to $\epsilon$, then we pass to the limit as $\omega^{\epsilon}$ tends to $\omega$. Property (8) is a regularity property and can be deduced as usual in the regularity theory for the Stokes equations, since $\mathbf{u}=\mathbf{v}-\mathbf{v}_{ \pm}$, along with some pressure function $\tau \in L_{l o c}^{2}(\Omega)$, is a weak solution of the Stokes equation $\nu \Delta \mathbf{u}=\nabla \tau+\mathbf{f}$, in the domain $\Omega_{t}^{c}$ for any sufficiently large $t$, where $\mathbf{f}:=\rho(\mathbf{v} \cdot \nabla) \mathbf{v}$. Because $\rho \in L^{\infty}(\Omega)$ one can verify that $\mathbf{f} \in L^{2}\left(\Omega_{t}\right)$ by a boot strap argument and Sobolev embedding theorems and so $\mathbf{u} \in W^{2,2}\left(\Omega_{t}\right)$; cf. [3, Lemma XI.4.1] where the homogeneous case $(\rho \equiv 1)$ is treated or see [4] for a complete proof. Regarding (9), from $\psi-\psi_{-} \in W^{2,2}\left(\Omega_{t}^{c}\right)$ we have $\lim _{x \rightarrow-\infty}\left\|\psi(x, \cdot)-\psi_{-}\right\|_{C_{b}\left(\Sigma_{-}\right)}=0$, then $\psi$ is bounded in $\Omega_{-}$and given an arbitrary $\epsilon>0$ there exists a $s>0$ such that $|x| \geq s$ implies $\left|\rho(x, y)-\rho_{-}(y)\right|=\left|\omega(\psi(x, y))-\omega\left(\psi_{-}(y)\right)\right|<\epsilon$ for all $y \in \Sigma_{-}$, since $\omega$ is locally uniformly continuous. The flux of $\mathbf{v}$ in $\Omega_{ \pm}$is equal to $\alpha_{ \pm}$because $\mathbf{v}=\mathbf{u}+\mathbf{a}$ with $\mathbf{u} \in \mathbf{V}$. Indeed, the flux of $\mathbf{u}$ in $\Omega_{ \pm}$is equal to zero and the flux of $\mathbf{a}$ in $\Omega_{ \pm}$is equal to $\alpha_{ \pm}$, since $\mathbf{a}$ coincides with $\mathbf{v}_{ \pm}$at large distances and $\nabla \cdot \mathbf{a}=0$. To prove (10) we have

$$
\begin{aligned}
\beta_{ \pm} & :=\int_{\Sigma_{ \pm}} \rho \mathbf{v} \cdot \mathbf{n}_{ \pm}= \pm \int_{-1}^{1}\left(\rho v_{1}\right)(x, y) d y= \pm \int_{-1}^{1}\left(\omega(\psi) \psi_{y}(x, y)\right) d y \\
& = \pm\left(-\int_{-1}^{1} \partial_{y}\left(\int_{0}^{\psi(x, y)} \omega(s) d s\right) d y\right) \\
& = \pm\left(\int_{0}^{\psi(x,-1)} \omega(s) d s-\int_{0}^{\psi(x, 1)} \omega(s) d s\right) \\
& = \pm \int_{\psi(x, 1)}^{\psi(x,-1)} \omega(s) d s= \pm \int_{\alpha_{ \pm}}^{0} \omega(s) d s= \pm \int_{\alpha_{ \pm}}^{0} \rho_{-}\left(\psi_{-}^{-1}(s)\right) d s .
\end{aligned}
$$

The condition $\|\rho\|_{C_{b}(\Omega)}$ is attained, as we mentioned above, by choosing $\omega$ satisfying also $\|\omega\|_{C_{b}(\mathbb{R})} \leq l$. Finally, we have (11) by the following steps: 


$$
\begin{aligned}
\left\|\nabla\left(\mathbf{v}-\mathbf{v}_{ \pm}\right)\right\|_{L^{2}\left(\Omega_{ \pm}\right)} & =\left\|\nabla\left(\mathbf{a}-\mathbf{v}_{ \pm}\right)\right\|_{L^{2}\left(\Omega_{ \pm}\right)}+\|\nabla \mathbf{u}\|_{L^{2}\left(\Omega_{ \pm}\right)} \\
& =\left\|\nabla\left(\mathbf{a}-\mathbf{v}_{ \pm}\right)\right\|_{L^{2}\left(\Omega_{ \pm, t}\right)}+\|\nabla \mathbf{u}\|_{L^{2}(\Omega)} \\
& \leq C\left|\alpha_{ \pm}\right|+\|\nabla \mathbf{u}\|_{L^{2}(\Omega)} \leq C\left|\alpha_{ \pm}\right|+C \frac{\left|\alpha_{ \pm}\right|\left(\nu+\left|\alpha_{ \pm}\right| l\right)}{\nu-c\left|\alpha_{ \pm}\right| l}
\end{aligned}
$$

where we used (13) and other estimates one can obtain to control $\|\nabla \mathbf{u}\|_{L^{2}(\Omega)}$, as indicated above. Analogously, one finds $\|\mathbf{v}\|_{L^{2}\left(\Omega_{0}\right)} \leq C\left|\alpha_{ \pm}\right|+C \frac{\left|\alpha_{ \pm}\right|\left(\nu+\left|\alpha_{ \pm}\right| l\right)}{\nu-c\left|\alpha_{ \pm}\right| l}$.

4. The density-dependent Leray problem: Discontinuous case. In this section we briefly describe the approximating scheme to achieve Theorem 2. For a rigorous and complete proof and more details we refer the reader to the forthcoming paper [5]. In [4] we also give a preliminary version of this result.

Our approximating scheme relies on an appropriate mollification of the data $\rho_{-}, \mathbf{v}_{-}$ and, at the approximated level, on the streamline function formulation. Let $\epsilon>0$ be arbitrary but sufficiently small. Extend $\rho_{-}$and $\psi_{-}$to $(-\infty, \infty)$ by zero outside $\Sigma_{-}$and let $\rho_{-}^{\epsilon}=\rho_{-} * m^{\epsilon}$ and $\psi_{-}^{\epsilon}=\psi_{-} * m^{\epsilon}$ be standard mollifications of these extended functions, i.e. * stands for convolution of functions on the real line and $m^{\epsilon}(y)=\epsilon^{-1} m(y / \epsilon)$, where $m$ is a positive smooth function with support in $(-1,1)$ and such that $\int_{-\infty}^{\infty} m(y) d y=1$. Next, let $\omega^{\epsilon}$ be a smooth function from $\mathbb{R}$ to $\mathbb{R}$ such that

$$
\left\|\omega^{\epsilon}\right\|_{C_{b}(\mathbb{R})} \leq l
$$

and

$$
\omega^{\epsilon}\left(\psi_{-}^{\epsilon}(y)\right)=\rho_{-}^{\epsilon}(y) \text { for } y \in \Sigma_{-}^{\epsilon}, \Sigma_{-}^{\epsilon}:=(-1+\epsilon, 1-\epsilon), .
$$

Notice that condition (19) on $\omega^{\epsilon}$ is reasonably imposed, since $\psi_{-}^{\epsilon}$ is an injective function. In fact, $\psi_{-}^{\epsilon}$ is a decreasing function since $\theta_{-}$is a strictly positive function on $\Sigma_{-}$and $m^{\epsilon}$ is also a strictly positive function on its support, i.e. on the interval $(-\epsilon, \epsilon)$. Thus we can write

$$
\omega^{\epsilon}(s)=\rho_{-}\left(\left(\psi_{-}^{\epsilon}\right)^{-1}(s)\right), \quad \text { for } s \in\left(\psi_{-}^{\epsilon}(1-\epsilon), \psi_{-}^{\epsilon}(-1+\epsilon)\right) .
$$

Outside the set $\left(\psi_{-}^{\epsilon}(1-\epsilon), \psi_{-}^{\epsilon}(-1+\epsilon)\right)$ we take $\omega^{\epsilon}$ arbitrary but smooth (say, $\left.C^{\infty}\right)$ and satisfying (18) for all sufficiently small $\epsilon>0$.

Our idea is to obtain an approximate solution $\left(\rho^{\epsilon}, \mathbf{v}^{\epsilon}\right)$ to our Problem (1)-(4) of the type $\left(\rho^{\epsilon}, \mathbf{v}^{\epsilon}\right)=\left(\omega^{\epsilon}\left(\psi^{\epsilon}\right), \nabla^{\perp} \psi^{\epsilon}\right)$ and recover an exact solution $(\rho, \mathbf{v})$ by taking the limit of $\left(\rho^{\epsilon}, \mathbf{v}^{\epsilon}\right)$ as $\epsilon$ goes to zero. From Theorem 3 there is a solution $\mathbf{u}^{\epsilon}=\nabla^{\perp} \psi^{\epsilon}-\mathbf{a} \in \mathbf{V}$, $\psi^{\epsilon} \in \mathbf{H}_{2, l o c}(\bar{\Omega})$ of the following variational problem:

$$
\nu \int_{\Omega} \nabla\left(\mathbf{u}^{\epsilon}+\mathbf{a}\right) \cdot \nabla \boldsymbol{\Phi} d x=\int_{\Omega} \omega^{\epsilon}\left(\psi^{\epsilon}\right)\left(\left(\mathbf{u}^{\epsilon}+\mathbf{a}\right) \cdot \nabla \boldsymbol{\Phi}\right) \cdot\left(\mathbf{u}^{\epsilon}+\mathbf{a}\right) d x, \forall \boldsymbol{\Phi} \in \mathcal{V}
$$

In view of (18) and the estimates indicated in the previous section, there exists a pair $(\rho, \mathbf{u})$ in $L^{\infty}(\Omega) \times \mathbf{V}$ such that, up to some subsequence of $\epsilon \rightarrow 0,\left(\mathbf{u}^{\epsilon}\right)$ converges to $\mathbf{u}$ in the weak topology of $\mathbf{V}$ and $\rho^{\epsilon}$ converges to $\rho$ in the weak-* topology of $L^{\infty}(\Omega)$ as $\epsilon \rightarrow 0$. Then it is possible to show that the pair $(\rho, \mathbf{v}), \mathbf{v}=\mathbf{u}+\mathbf{a}$, satisfies all the claims stated in Theorem $2[5,4]$. 


\section{References}

[1] C. J. Amick, Steady solutions of the Navier-Stokes equations in unbounded channels and pipes, Ann. Scuola Norm. Sup. Pisa Cl. Sci. (4) 4 (1977) 473-513.

[2] N. N. Frolov, Solvability of a boundary problem of motion of an inhomogeneous fluid, Mat. Zametki 53 (1993), 130-140.

[3] G. P. Galdi, An Introduction to the Mathematical Theory of the Navier-Stokes Equations, v. II, Springer-Verlag, 1994.

[4] F. Ammar-Khodja and M. M. Santos, Stationary solution of the Navier-Stokes equations for inhomogeneous incompressible fluids in $2 d$ domains with channels having bounded cross sections, Technical report \# 01/03, IMECC-UNICAMP, Brazil 2003.

[5] F. Ammar-Khodja and M. M. Santos, The 2d density-dependent Leray problem with a discontinuous density, in preparation, 2004.

[6] O. A. Ladyzhenskaya, Stationary motion of a viscous incompressible fluid in a pipe, Dokl. Akad. Nauk SSSR 124 (1959), 551-553.

[7] M. M. Santos, Stationary solution of the Navier-Stokes equations in a 2d bounded domain for incompressible flow with discontinuous density, ZAMP-Z. Angew. Math. Phys. 53 (2002), 661-675.

[8] R. Temam, Navier-Stokes equations. Theory and Numerical Analysis. Studies in Mathematics and its Applications 2. North-Holland, Amsterdam-New York-Oxford, 1979. 